\title{
Ultra High Energy Resolution EELS Map Employing an Aberration-corrected STEM Equipped with a Monochromator
}

\author{
Masaki Mukai ${ }^{1}$, Eiji Okunishi ${ }^{1}$, Masanori Ashino ${ }^{1}$, Kazuya Omoto ${ }^{1}$, Tomohisa Fukuda ${ }^{1}$, Akihiro Ikeda ${ }^{1}$, \\ Kazunori Somehara ${ }^{1}$, Toshikatsu Kaneyama ${ }^{1}$, Tomohiro Saitoh ${ }^{2}$, Tsukasa Hirayama ${ }^{2}$, Yuichi Ikuhara ${ }^{2,3}$ \\ 1. JEOL Ltd., 3-1-2 Musashino, Akishima, Tokyo 196-8558, Japan \\ 2. Japan Fine Ceramics Center, 2-4-1 Mutsuno, Atsuta-ku, Nagoya, 456-8587, Japan \\ 3. The University of Tokyo, 2-11-16 Yayoi, Bunkyo-ku, Tokyo 113-8656 Japan.
}

Scanning transmission electron microscope (STEM) equipped with an aberration corrector enables us to investigate the elemental analysis at atomic scale by electron energy loss spectroscopy (EELS) since the technology of the spherical aberration correction has improved the spatial resolution of the transmission electron microscope. [1, 2] Moreover, by installing a monochromated electron source to an aberration corrected electron microscope, the detailed electronic state analysis with the atomic resolution can be revealed with the better energy resolution. As shown in figure 1, we have developed an analytical electron microscope equipped with a monochromator (JEM-2400FCS), which is based on JEMARM200F equipped with a hexapole type spherical aberration corrector for STEM (CEOS GmbH) and a high resolution spectrometer of TridiemERS (GATAN Inc.).

The developed monochromator, located between the extraction anode of the $\mathrm{ZrO} / \mathrm{W}$ emitter and the accelerator, consists of two Wien-filters and an energy selection slit inserted between two filters. The width of the slit is selectable by the mechanical movement. This configuration is similar to the previous design. [3] The first Wien-filter produces a spectrum of the electron source at the slit with an energydispersion. After the energy selection by the slit, the second Wien-filter cancels the energy-dispersion and produces an achromatic and stigmatic focus at the exit of the monochromator. Due to this achromatic and stigmatic focus, the roundly symmetrical probe is produced on the specimen plane. The electro-static round lens of the accelerator is designed to optimize not only $200 \mathrm{kV}$ but also $60 \mathrm{kV}$ operations. Figure 2 shows the relatively normalized intensity profiles of the zero-loss peaks for the several widths of the slits obtained with a 0.1 seconds acquisition at $200 \mathrm{kV}$. The energy resolution of 36 $\mathrm{meV}$ was obtained with a 0.1 seconds acquisition using the slit of $0.25 \mu \mathrm{m}$.

We tested the EELS map in the low-loss region on a $\mathrm{TiO}_{2}$ (rutile) specimen obtained with a $146 \mathrm{meV}$ energy spread, a $112 \mathrm{pA}$ probe current, a $37.5 \mathrm{mrad}$ convergent angle and a 0.05 seconds acquisition in each pixel. Figure 3(a) shows the ADF signal image and Fig.3(b) shows the extracted low-loss spectra from the each rectangle area in Fig.3(a). Figure 3(c) show the EELS maps obtained with a $0.14 \mathrm{eV}$ energy window from 9 energy regions, which have differences of the intensity distribution. Especially, in 6 maps of energy-loss of less than $6 \mathrm{eV}$, the surface plasmon signal was detected from not only the surface area but the vacuum area beyond the edge of the specimen. These plasmon maps show the one of the advantages of a monochromator, which enables us to detect a clear low-loss spectrum without any deconvolution processings due to the sharp drop of the intensity distribution of the zero-loss peak.

The detailed results of the basic performance tests obtained at the accelerating voltage of $200 \mathrm{kV}$ and 60 $\mathrm{kV}$ will be reported at the meeting,

References:

[1] E. Okunishi et al.: Microscopy \& Microanalysis, vol.13 (supple.2), 1150-1151 (2006)

[2] D. A. Muller et al.: Science 319, 1073 (2008)

[3] M. Mukai et al.: Proceeding IMC17-I1.2 (2010) 


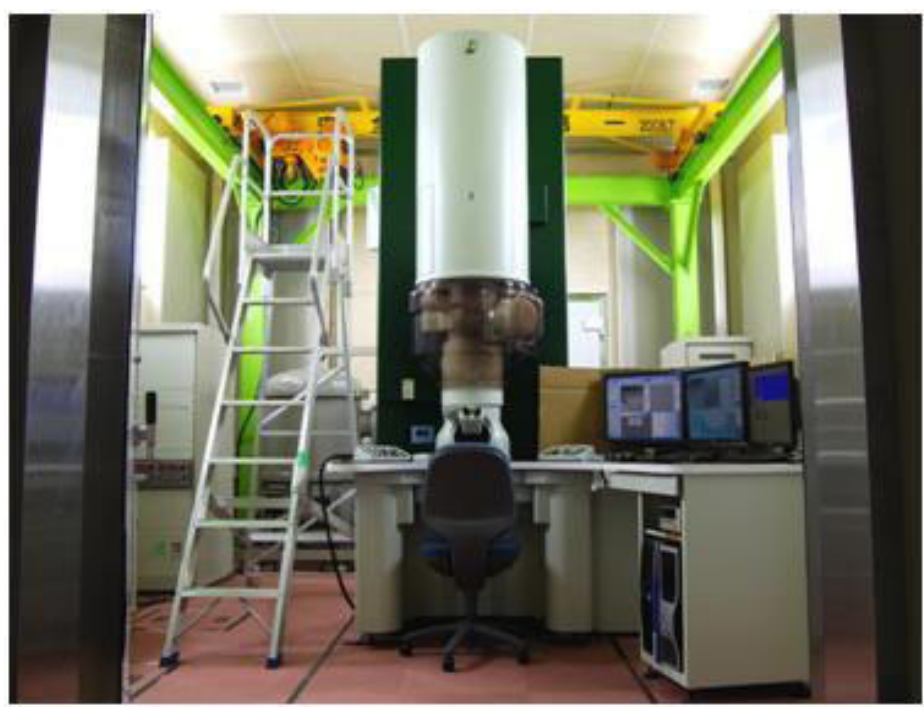

Figure 1.The appearance of JEM-ARM200F equipped with a monochromator (JEM-2400FCS)

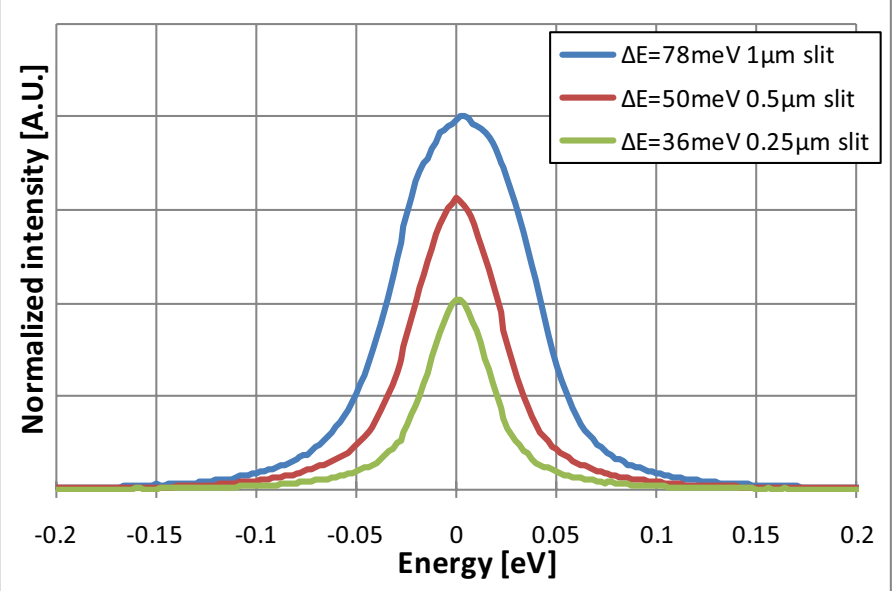

Figure 2. Relatively normalized intensity profiles of zero-loss peaks for the slit of $0.25 \mu \mathrm{m}, 0.5 \mu \mathrm{m}$ and $1 \mu \mathrm{m}$ obtained with 0.1 seconds acquisition at $200 \mathrm{kV}$

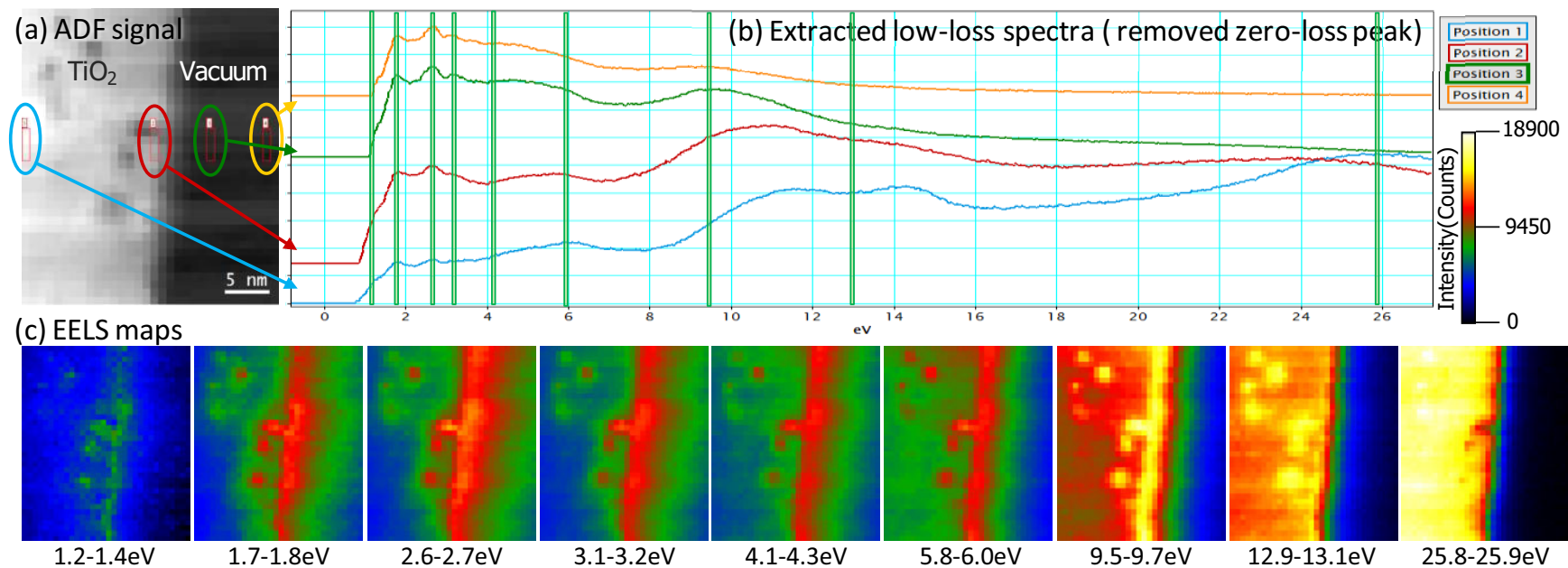

Figure 3. (a) ADF signal image of $\mathrm{TiO}_{2}$ taken at $200 \mathrm{kV}$ with an energy spread of $146 \mathrm{meV}$

(b) Extracted low-loss spectra integrated from each rectangle area in Fig.3(a)

(c) EELS maps obtained with a $0.14 \mathrm{eV}$ energy window from 9 energy regions 